\title{
Equipamentos de proteção individual e a adesão do conhecimento dos profissionais e acadêmicos: revisão integrativa
}

\author{
Personal protective equipment and the adherence of professional and academic \\ knowledge: an integrative review
}

\author{
Equipo de protección personal y adhesión al conocimiento profesional y académico: una \\ revisión integradora
}

André Pessoa Silva de Bastos ${ }^{1}$, Madelyne Alice Ramos Brito ${ }^{1}$, Jéssica de Lima Rego Ferreira ${ }^{1}$, José Guilherme de Oliveira Rodrigues Ferreira ${ }^{1}$, Vitória Stéfanny Cunha Araujo ${ }^{1}$, Hendrix Marçal Carvalho Val $^{1}$, Alba Angélica Nunes Mouta ${ }^{2 \star}$, Yuri Dias Mâcedo Campelo ${ }^{1}$, Joilson Ramos de Jesus ${ }^{1}$, Renata Paula Lima Beltrão ${ }^{1,2}$.

\section{RESUMO}

Objetivo: Verificar através de uma revisão de literatura os riscos ocupacionais devido à negligência e as dificuldades do uso de Equipamentos de Proteção Individual (EPI) por profissionais e estudantes da saúde, bem como descrever brevemente o entendimento e a real utilização por parte destes no Brasil. Métodos: Revisão de Literatura Integrativa, cujo artigos foram buscados nas bases de Dados SCIELO, MEDLINE e LILACS. Resultados: Concluiu-se que prevalece a negligência das normas de biossegurança em locais de assistência à saúde, principalmente devido a fatores como o não entendimento da importância dos EPI, a escassez de recursos financeiros e pelo simples comodismo, associado ao desinteresse e a autoconfiança. São evidenciados altos números de acidentes com exposição à materiais biológicos, perfurocortantes e possibilidade de infecções cruzadas relacionadas a essa má prática. Cabe a realização de programas de conscientização continuados sobre o uso dos EPI para profissionais e alunos da área da saúde. Considerações finais: Foi observado nos artigos da literatura uma significativa negligência dos profissionais quanto ao uso dos EPI a fatores como a falta de conhecimento e capacitação, a ausência de recursos necessários, o comodismo gerado pela autoconfiança de procedimentos rotineiros e até mesmo o desinteresse.

Palavras-chave: Equipamentos de proteção individual, Saúde do trabalhador, Pessoal de saúde.

\section{ABSTRACT}

Objective: To verify through a literature review the occupational risks due to negligence and the difficulties in the use of Personal Protective Equipment (PPE) by health professionals and students, as well as to briefly describe their understanding and real use in Brazil. Methods: Integrative Literature Review, whose articles were searched in the SCIELO, MEDLINE and LILACS databases. Results: It was concluded that the neglect of biosafety rules in health care places prevails, mainly due to factors such as the lack of understanding of the importance of PPE, the scarcity of financial resources and the simple convenience, associated with disinterest and self-confidence. High numbers of accidents with exposure to biological materials, sharps and the possibility of cross infections related to this bad practice are evidenced. Continuing awareness programs on the use of PPE for health professionals and students should be carried out. Final considerations: It was observed in the literature articles a significant negligence of professionals regarding the use of PPE for factors such as the lack of knowledge and training, the lack of necessary resources, the comfort generated by the self-confidence of routine procedures and even disinterest.

Key words: Personal protective equipment, Occupational health, Health personnel.

${ }^{1}$ Instituto de Educação Superior do Vale do Parnaíba (IEVASP). Parnaíba - PI.

2Universidade Federal do Piauí (UFPI).Parnaíba - PI. `E-mail: angelicanmouta@gmail.com 


\section{RESUMEN}

Objetivo: Para verificar a través de una revisión bibliográfica los riesgos laborales debido a negligencia y las dificultades en el uso de Equipos de Protección Personal (PPE) por parte de profesionales de la salud y estudiantes, así como describir brevemente su comprensión y uso real en Brasil. Métodos: Revisión Integral de Literatura, cuyos artículos fueron buscados en las bases de datos SCIELO, MEDLINE y LILACS. Resultados: Se concluyó que prevalece la negligencia de las reglas de bioseguridad en los lugares de atención médica, principalmente debido a factores como la falta de comprensión de la importancia del EPP, la escasez de recursos financieros y la simple conveniencia, asociada con el desinterés y la falta de interés. confianza en uno mismo. Se evidencia un alto número de accidentes con exposición a materiales biológicos, objetos punzantes y la posibilidad de infecciones cruzadas relacionadas con esta mala práctica. Deben llevarse a cabo programas de sensibilización continua sobre el uso de EPP para profesionales de la salud y estudiantes. Consideraciones finales: Se observó en los artículos de la literatura una negligencia significativa de los profesionales con respecto al uso de EPP por factores como la falta de conocimiento y capacitación, la falta de recursos necesarios, la comodidad generada por la autoconfianza de los procedimientos de rutina e incluso el desinterés.

Palabras clave: Equipo de protección personal, Salud laboral, Personal de salud.

\section{INTRODUÇÃO}

Os Equipamentos de proteção individual (EPI) são dispositivos de uso individual que são utilizados por diversos profissionais, dentre eles os profissionais da saúde, que servem para prevenir riscos que podem ameaçar a saúde dos trabalhadores durante a execução das atividades laborais. Esses dispositivos atuam como medida protetivas para a redução dos riscos biológicos, físicos e químicos. No âmbito da saúde, são exemplos de EPI os jalecos, as luvas, as máscaras, os gorros, os uniformes, os óculos de acrílico, os protetores faciais, os aventais e as botas ou sapatos fechados impermeáveis (RISSO M, RIBEIRO MC, 2003).

Os riscos de acidentes com materiais biológicos são os mais frequentes para profissionais da saúde, esses acidentes ocorrem quando existe o contato do trabalhador com sangue e/ou outros fluidos do paciente, que acontecem por inoculação percutânea ou por contato direto com pele e/ou mucosa durante os acidentes com perfurocortantes. Um grave problema é que geralmente quando ocorrem esses acidentes, os profissionais tendem a não os notificar, por receio ou por o processo de notificação ser demorado e exigir a realização de testes e o acompanhamento médico (SARDEIRO TL, et al., 2019).

Existem mais de vinte doenças que podem ser transmitidas por contato com os fluidos dos pacientes, dentre elas, as principais são Síndrome da Imunodeficiência Humana (AIDS), causado pelo vírus da Imunodeficiência Humana (HIV), hepatite B, causada pelo vírus da Hepatite B (HBV) e hepatite C causado pelo vírus da Hepatite $\mathrm{C}(\mathrm{HCV})$.

O risco de ocorrer a soroconversão nessas doenças quando o paciente-fonte tem sorologia positiva para uma das doenças anteriormente citada é maior na hepatite $B$, sendo de 6 a $30 \%$; já na hepatite $C$ chega a 0,5 a $2 \%$ e na AIDS é de $0,3 \%$.

Caso o resultado do teste rápido seja positivo, o acidentado deve iniciar quimioprofilaxia para a doença em específico e continuar com o seguimento clínico, caso seja negativo, deve continuar com 0 acompanhamento médico. $\mathrm{O}$ acompanhamento deve ser ambulatorial e dura de 6 a 12 meses, o profissional deve receber atendimento no momento do acidente, com 1 mês, com 3 meses, com 6 meses e com 12 meses, a depender do caso em questão.

Caso não ocorra soroconversão, o paciente é liberado do serviço de saúde, caso ocorra a soroconversão, o paciente deve ser encaminhado para atendimento com médico infectologista (CAIXETA RB, BARBOSABRANCO A, 2005; DIAS MAC, et al., 2012; GUIMARÃES HV, et al., 2011; RIBEIRO S, et al., 2009; RIFFEL FA, 2015; SILVA TR, et al., 2010). 
No Brasil, a Norma Regulamentadora 32 estabelece as diretrizes básicas para a implementação de medidas de proteção à segurança de trabalhadores em serviço de saúde; e dispõe que é de responsabilidade das instituições de saúde a disponibilização de EPI, sem ônus para os profissionais, e que os mesmos não devem realizar o seu uso fora das atividades ocupacionais. A preocupação central seria a construção de ambientes saudáveis para os procedimentos técnicos, alertando que a desobediência à essa normativa pode determinar ameaças adicionais às atividades laborais (KAISER TL, et al., 2016).

A problemática está além da disponibilidade e do uso dos EPI, visto que muitos profissionais não sabem manipular esses EPI, reforçando que o mau uso dessas ferramentas implica em problemas à saúde, aumentando o risco de infecções cruzadas, sendo determinantes de prejuízos ao paciente, aos profissionais e à instituição de saúde, tanto financeiros quanto na qualidade do serviço prestado (LEITE CJ, et al., 2019).

Ademais, como o demonstrado nos estudos de Rodrigues PS, et al., (2017) é necessário fazer o descarte adequado dos $\mathrm{EPI}$, em locais específicos e embalagens protetores que isolem o material do ambiente, bem como promover o monitoramento e a sua autoclavação, que consiste basicamente na prática de esterilizar o material a ser rejeitado.

O objetivo do estudo é observar através de uma revisão integrativa sobre os riscos ocupacionais devido à negligência e as dificuldades do uso de EPI, avaliando o impacto que o mau uso desses recursos gera nos profissionais e estudantes da saúde. Também tem como objetivo, falar sobre a importância dos EPI, as dificuldades no uso e a resistência de seu uso por profissionais e estudantes da saúde.

\section{MÉTODOS}

Trata-se de uma revisão de literatura integrativa sobre os EPI, visando avaliar a adesão e o conhecimento dos profissionais e estudantes da área da saúde sobre a importância e o uso correto desses materiais durante as atividades práticas e ambiente de trabalho.

A busca dos artigos foi feita nas seguintes bases de dados Scientific Electronic Library Online (SciELO), Medical Literature Analysis and Retrievel System Online (Medline) e Literatura Latino-Americana e do Caribe em Ciências da Saúde (LILACS), utilizando os descritores "Equipamentos de proteção individual", "Saúde do trabalhador" e "Pessoal da saúde".

Ao utilizar os três descritores na base MEDLINE não foi encontrado nenhum resultado, em seguida, a busca foi feita utilizando os descritores associados em pares "pessoal da saúde" e "saúde do trabalhador" foram encontrados dois artigos, as outras duas duplas de pares não resultaram nenhum resultado.

$\mathrm{Na}$ base SCIELO, ao utilizar os três descritores em associação foi encontrado um artigo, ao usa o par equipamento de proteção individual e saúde do trabalhador foram encontrados trinta artigos, utilizando o par "equipamento de proteção individual" e "pessoal da saúde" foram encontrados cinco artigos e o par "pessoal da saúde" e "saúde do trabalhador" foram encontrados cento e cinquenta e quatro artigos.

Já na base LILACS, utilizando os três descritores em associação não obteve resultados, utilizando a associação equipamento de proteção individual e saúde do trabalhador foram encontrados cinco artigos e a associação equipamento de proteção individual e pessoal da saúde não obteve resultado e saúde do trabalhador e pessoal da saúde também não obteve resultados.

Dos cento e noventa e sete artigos encontrados na busca inicial, foi feita inicialmente a leitura do título desses artigos, os que não se enquadravam no tema de estudo foram descartados, sendo eliminados oitenta e oito documentos por esse motivo. Sobrando um total de cento e nove para a leitura dos resumos, sendo eliminados oitenta e três porque não tratavam sobre o tema de estudo.

Ao final, sobraram vinte e seis artigos para a leitura integral para a realização da revisão de literatura, também foi acrescentando o livro Biossegurança em odontologia e ambientes de saúde e um guia prático para avaliação e condutas frente aos acidentes com exposição a fluidos biológicos ao estudo. Com o intuito de proporcionar maior visibilidade e objetividade dos dados obtidos no livro e nos vinte e seis artigos selecionados, construímos a tabela apresentada abaixo (Tabela 1). 
Os critérios de inclusão foram: artigos científicos disponibilizados de forma gratuita e integral, que fossem pesquisas em seres humanos e que fossem publicados entre 2000 e 2020. Os critérios de exclusão foram: materiais que não fossem artigos científicos e estudos que não estivessem de acordo com o tema selecionado.

Figura 1 - Quantidade de utilizados para a realização da revisão.

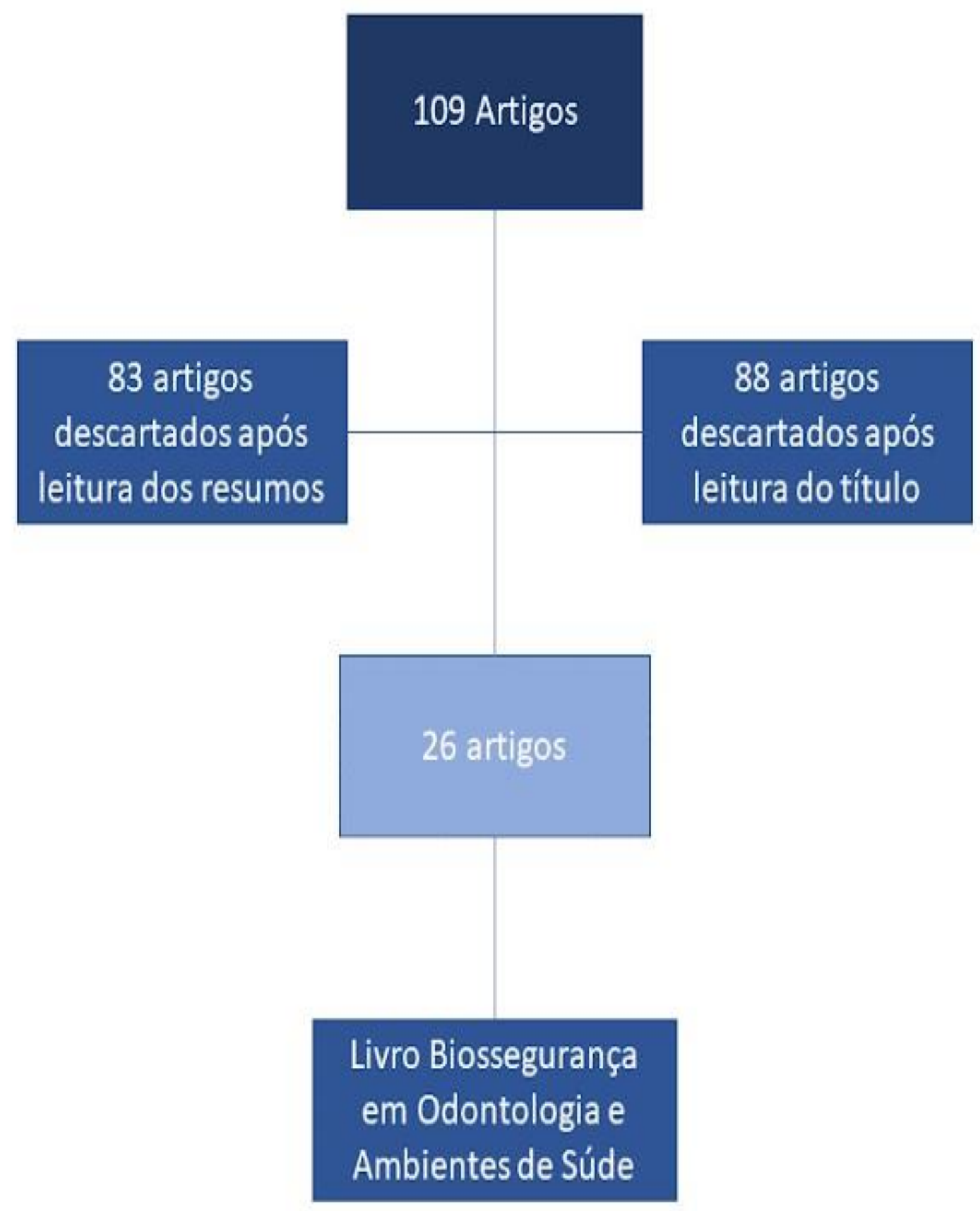

Fonte: Bastos APS, et al., 2020. 
Quadro 1 - Distribuição dos dados da pesquisa em relação ao ano de publicação, periódico em que foram publicados ou nome do livro e objetivo da publicação.

\begin{tabular}{|c|c|c|c|}
\hline Obra & Ano & Periódico do Artigo ou Livro & Objetivo \\
\hline 1 & 2009 & $\begin{array}{l}\text { Livro Biossegurança em odontologia e } \\
\text { ambientes de saúde. }\end{array}$ & $\begin{array}{l}\text { Apresentar as normas de biossegurança impostas nos ambientes de saúde e sua importância na } \\
\text { prevenção de acidentes com perfurocortantes e materiais biológicos. }\end{array}$ \\
\hline 2 & 2016 & SaBios-Revista de Saúde e Biologia. & $\begin{array}{l}\text { Avaliar a contaminação microbiana em jalecos de estudantes da área da saúde e a importância do uso } \\
\text { correto dos EPIs. }\end{array}$ \\
\hline 3 & 2019 & Revista ABENO & $\begin{array}{l}\text { Revisar a literatura científica sobre a importância da desinfecção no ambiente de trabalho dos laboratórios } \\
\text { quanto nos consultórios, e assim, controlar a via de transmissão cruzada. }\end{array}$ \\
\hline 4 & 2019 & REVISA & $\begin{array}{l}\text { Compreender o entendimento dos profissionais de saúde básica de um município de Goiás, no que se } \\
\text { refere à utilização de equipamentos de proteção individual, focado nos técnicos de enfermagem e } \\
\text { enfermeiros. }\end{array}$ \\
\hline 5 & 2017 & $\begin{array}{l}\text { Revista Online de pesquisa "Cuidado é } \\
\text { Fundamental" }\end{array}$ & $\begin{array}{l}\text { Identificar os fatores que expõem os trabalhadores de enfermagem de UTI aos acidentes por } \\
\text { perfurocortantes durante a sua manipulação e descarte. }\end{array}$ \\
\hline 6 & 2017 & $\begin{array}{l}\text { Revista Brasileira de Medicina do } \\
\text { Trabalho }\end{array}$ & $\begin{array}{l}\text { Analisar os fatores associados e determinar a frequência de adesão ao uso EPIs por profissionais de } \\
\text { saúde que sofreram acidentes ocupacionais com material biológico no estado do Maranhão. }\end{array}$ \\
\hline 7 & 2015 & $\begin{array}{l}\text { Trabalho de Conclusão de Curso de } \\
\text { Pós-graduação em enfermagem do } \\
\text { trabalho }\end{array}$ & $\begin{array}{l}\text { Discutir a resistência ao uso dos Equipamentos de Proteção Individual (EPIs) pela equipe de enfermagem } \\
\text { no ambiente hospitalar, e relacionar as causas que levam estes profissionais a não aderirem às } \\
\text { precauções de segurança. }\end{array}$ \\
\hline 8 & 2017 & Escola de Enfermagem Ana Nery & $\begin{array}{l}\text { Identificar a prevalência de acidentes ocupacionais entre profissionais de enfermagem atuantes em } \\
\text { setores críticos de um pronto-socorro e aprender a vivência profissional dentre os acidentados. }\end{array}$ \\
\hline 9 & 2019 & $\begin{array}{l}\text { Monografia apresentada ao curso de } \\
\text { Graduação em enfermagem FAEMA }\end{array}$ & $\begin{array}{l}\text { Trata-se de uma pesquisa de campo com abordagem quantitativa e qualitativa, tendo como objetivo } \\
\text { analisar a adesão do uso de Equipamentos de Proteção Individual na equipe de enfermagem, de um } \\
\text { hospital de médio porte na cidade de Monte Negro- RO. }\end{array}$ \\
\hline 10 & 2018 & $\begin{array}{l}\text { Revista Online de pesquisa "Cuidado é } \\
\text { Fundamental" }\end{array}$ & $\begin{array}{l}\text { Conhecer e analisar a produção científica em relação a biossegurança e os fatores de risco vivenciados } \\
\text { pelo enfermeiro no seu contexto hospitalar. }\end{array}$ \\
\hline 11 & 2015 & Revista Trabalho, Educação e Saúde & $\begin{array}{l}\text { Identificar as concepções de biossegurança de docentes do ensino técnico de enfermagem que atuam } \\
\text { em instituições vinculadas à Rede Federal de Educação Profissional e Tecnológica de um estado do Sul } \\
\text { do Brasil. }\end{array}$ \\
\hline 12 & 2015 & Revista CogitareEnferm & $\begin{array}{l}\text { Trata-se de um estudo observacional, descritivo, transversal com abordagem quantitativa, desenvolvido } \\
\text { em um hospital público com atendimento de alta complexidade em uma cidade do norte do Paraná. }\end{array}$ \\
\hline 13 & 2017 & $\begin{array}{l}\text { Revista Online de pesquisa "Cuidado é } \\
\text { Fundamental" }\end{array}$ & $\begin{array}{l}\text { Identificar os fatores que expõe os trabalhadores de enfermagem de UTI aos acidentes por } \\
\text { perfurocortantes durante a manipulação e descarte. }\end{array}$ \\
\hline
\end{tabular}

REAS/EJCH | Vol.Sup.n.53 | e3764 | DOI: https://doi.org/10.25248/reas.e3764.2020 Página $\mathbf{5}$ de $\mathbf{1 0}$ 


\begin{tabular}{|c|c|c|c|}
\hline Obra & Ano & Periódico do Artigo ou Livro & Objetivo \\
\hline 14 & 2018 & $\begin{array}{l}\text { Revista Online de pesquisa "Cuidado é } \\
\text { Fundamental" }\end{array}$ & $\begin{array}{l}\text { Identificar motivações para mudança nas ações dos profissionais, após acidente ocupacional e discutir } \\
\text { mudanças no processo de trabalho na perspectiva da saúde do trabalhador. }\end{array}$ \\
\hline 15 & 2015 & RevistaPan-Amazônica de Saúde & $\begin{array}{l}\text { Analisar o conhecimento e a aplicação das normas de biossegurança entre acadêmicos de Odontologia } \\
\text { da Universidade Federal do Pará, a fim de verificar as divergências entre dois diferentes momentos da } \\
\text { formação profissional e superar as deficiências detectadas. }\end{array}$ \\
\hline 16 & 2019 & $\begin{array}{l}\text { Revista da Escola de Enfermagem da } \\
\text { USP }\end{array}$ & $\begin{array}{l}\text { Analisar o perfil de profissionais da saúde que sofreram acidentes com exposição a materiais biológicos } \\
\text { durante } 2006 \text { e } 2016 \text { no Estado de Goiás. }\end{array}$ \\
\hline 17 & 2016 & Revista de Enfermagem da UERJ & $\begin{array}{l}\text { Identificar os modos de exposição que os enfermeiros estão expostos na Atenção Primária a Saúde na } \\
\text { cidade de Goiânia (GO) }\end{array}$ \\
\hline 18 & 2010 & Revista Nursing & $\begin{array}{l}\text { Analisa o impacto do uso de luvas na redução dos riscos de acidentes por materiais biológicos e a adesão } \\
\text { dos profissionais de enfermagem ao seu uso. }\end{array}$ \\
\hline 19 & 2011 & $\begin{array}{l}\text { Revista Latino Americana de } \\
\text { Enfermagem }\end{array}$ & $\begin{array}{l}\text { Pesquisa realizada com } 15 \text { enfermeiros, buscando avaliar a adesão dos equipamentos de proteção } \\
\text { individual. }\end{array}$ \\
\hline 20 & 2016 & Revista Trabalho, Educação e Saúde & $\begin{array}{l}\text { Analisar a prática de biossegurança entre os enfermeiros que atuam como docentes em uma instituição } \\
\text { Federal em um Hospital na região Sul do país. }\end{array}$ \\
\hline 21 & 2005 & Caderno de Saúde Pública & $\begin{array}{l}\text { Estudo realizado em seis hospitais do distrito Federal com o objetivo de entrevistar os profissionais de } \\
\text { saúde sobre o uso de EPI e a ocorrência de acidentes de trabalho. }\end{array}$ \\
\hline 22 & 2012 & Medicina (Ribeirão Preto) & Análise retrospectiva dos casos de acidentes ocupacionais que envolvem materiais biológicos. \\
\hline 23 & 2011 & $\begin{array}{l}\text { Guia Sobre conduta em casos de } \\
\text { acidentes perfurocortantes }\end{array}$ & $\begin{array}{l}\text { Faz um levantamento sobre o passo-a-passo que os profissionais devem seguir em caso de acidentes } \\
\text { com materiais perfurocortantes. }\end{array}$ \\
\hline 24 & 2009 & Cogitare Enfermagem & $\begin{array}{l}\text { Análise dos acidentes de trabalho que ocorreram em um Hospital com a equipe de profissionais de } \\
\text { enfermagem e de acadêmicos de enfermagem. }\end{array}$ \\
\hline 25 & 2015 & Saúde Integrada - Biomedicina & $\begin{array}{l}\text { Pesquisa realizada no Hospital de Santa Rosa - RS, com o objetivo de entrevistar profissionais que } \\
\text { sofreram acidentes de trabalho. }\end{array}$ \\
\hline 26 & 2010 & Revista Gaúcha de Enfermagem & $\begin{array}{l}\text { Análise das fichas de notificação dos acidentes de trabalho com materiais perfurocortantes entre os } \\
\text { profissionais de enfermagem de um hospital universitário. }\end{array}$ \\
\hline 27 & 2009 & Cienc Cuid Saude & $\begin{array}{l}\text { Estudo realizado em hospital particular com o objetivo de avaliar a incidência de acidentes com materiais } \\
\text { perfurocortantes com os acadêmicos de cursos da área da saúde. }\end{array}$ \\
\hline 28 & 2008 & Rev. odonto ciênc. & $\begin{array}{l}\text { Aplicação de questionário semi-estruturado para o uso de EPI e a ocorrência de acidentes de trabalho } \\
\text { com os profissionais do hospital. }\end{array}$ \\
\hline
\end{tabular}

Fonte: Bastos APS, et al., 2020.

REAS/EJCH | Vol.Sup.n.53 | e3764 | DOI: https://doi.org/10.25248/reas.e3764.2020 Página $\mathbf{6}$ de $\mathbf{1 0}$ 


\section{RESULTADOS E DISCUSSÃO}

Nos artigos analisados percebe-se uma concordância de que alguns fatores de risco estão presentes no cotidiano de trabalho dos profissionais e estudantes da saúde, em proporções distintas, a depender do setor e instituição de prestação de serviço. A carência de recursos materiais, déficit no dimensionamento de pessoal, falha no conhecimento e capacitações frente às questões de biossegurança são fatores potencializadores de risco dentro dos cenários de atuação da saúde, interferindo no bem estar psicossocial, físico e moral.

A biossegurança é definida pela Agência Nacional de Vigilância Sanitária (ANVISA) como o conjunto de medidas de ações destinadas a prevenir, reduzir e/ou eliminar riscos para a saúde dos seres humanos. Acredita-se que a biossegurança no contexto dos trabalhadores da área da saúde é um desafio, pois, com uma maior experiência adquirida com o tempo na profissão, maior é a tendência de fazer um trabalho mecanizado e fluido, e assim, as normas de segurança como a utilização de equipamentos de proteção, acabam sendo frequentemente negligenciadas, embora o trabalhador saiba da importância destas. Por isso, muitas pesquisas sobre o assunto abordam a educação continuada na saúde como solução, já que esta serviria como ferramenta de reflexão e consequente mudança de hábitos e comportamentos dentro do contexto hospitalar, minimizando acidentes de exposição a materiais biológicos e perfurocortantes (ANDRADE GB, et al., 2018).

A resistência ao uso dos EPI por parte dos profissionais é uma realidade, os principais motivos para esse comportamento seriam a autoconfiança proveniente da experiência, a sobrecarga de trabalho, 0 desconhecimento parcial dos riscos a que estão susceptíveis, a perda de habilidade, o desconforto no uso e as falhas na vigilância da equipe de saúde e no processo educacional de orientação e aprendizagem. O uso de EPI não elimina os riscos de possíveis acidentes e de exposição a agente biológicos, mas ao serem utilizados de forma correta, diminuem substancialmente esses riscos. Também é importante destacar, que 0 uso desses equipamentos não elimina as medidas de higienização pessoal, como a desinfecção das mãos (DIAS ACB, et al., 2015; PINA E, 2010).

Em todo o mundo, existem cerca de 35 milhões de trabalhadores da área da saúde, dos quais 3 milhões já tiveram algum tipo de exposição percutânea ou foram expostos a vírus transmitidos pelo sangue devido acidentes com perfurocortantes. A exposição elevada ocorre principalmente por falta de direcionamento de políticas públicas, falta de aperfeiçoamento dos profissionais de saúde, desmotivação e sobrecarga de serviço, por isso a importância de as instituições de saúde ofertarem cursos de educação continuada em saúde (PEREIRA APDS, VERISSIMO TDC, 2019; SOUSA AFL, et al., 2016).

Em estudo conduzido por Rezende KCD (2016) que analisou a realização de diversos procedimentos que expunha o profissional ao risco de contaminação biológica, dentre eles: imunização, realização do pré-natal, teste do pezinho e exame colpocitológico, a falta do uso dos EPI foi apontado como o principal fator para a contaminação dos profissionais, bem como o uso incorretos desses utensílios, visto que nenhum deles protege os profissionais $100 \%$ do risco de contaminação e seu uso de forma incorreta pode diminuir ainda mais o grau de proteção.

Dentre os profissionais mais expostos ao risco de acidentes ocupacionais com materiais biológicos, estão os enfermeiros e os técnicos de enfermagem, porque são os profissionais que estão em contato mais próximo com os pacientes e que manipulam com maior frequência objetos perfurocortantes para a realização de procedimento, estando também em contato direto com os fluidos dos pacientes. Por esses motivos, esses profissionais são os que mais sofrem acidentes de trabalho envolvendo materiais biológicos e os que mais estão expostos a esses acidentes, mesmo utilizando os EPI (NEVES HCC, 2011).

Em uma pesquisa realizada no pronto-socorro do Hospital Regional de Ceilândia, uma cidade de grande porte do Distrito Federal, com amostra de 75 profissionais, sendo 14 enfermeiros e 61 técnicos de enfermagem, identificou-se uma maior frequência de acidentes em profissionais com idade superior a 40 anos, mesmo com grande experiência, estes admitiram negligenciar o uso de medidas de precaução. Todos os profissionais entrevistados relataram estar cientes dos riscos e das consequências envolvidas no acidente com material biológico, porém constatou-se que a maior parte deles não sabia como proceder (RODRIGUES PS, et al., 2010). 
Um levantamento estatístico realizado com uma equipe de enfermagem no Norte do Brasil, mostrou uma deficiência na capacitação destes profissionais, onde $65 \%$ afirmaram não ter capacitação sobre o uso devido de EPI, alegando desconhecimento sobre a maneira correta de utiliza-los e de descarta-los, e que não recebiam treinamento apropriado por parte da instituição em que trabalhavam (PEREIRA APDS, VERISSIMO TDC, 2019).

Ao se realizar um questionário entre os técnicos de enfermagem, sobre a funcionalidade dos EPI, nos estudos de Garcez GO, et al. (2019), 54\% dos entrevistados afirmam que os equipamentos protegem somente o profissional, enquanto $46 \%$ responderam que os equipamentos protegem o paciente e o profissional. Entre enfermeiros, $45 \%$ disseram que era apenas para proteção profissional e $55 \%$ que afirmaram que os EPI protegem paciente e profissional. Este estudo demonstra a não compreensão sobre a funcionalidade dos EPI nas classes investigadas, alertando sobre o desconhecimento da Norma Reguladora desses EPI, que são utilizados como instrumento de proteção para o profissional, logo, se os profissionais não tem ciência da importância e da utilidade desses objetos, é mais fácil que os utilizem de forma incorreta.

A temática da biossegurança não é abordada com tanta frequência nas diretrizes curriculares nacionais dos cursos de graduação na área da saúde, apresentando-se de forma bastante genérica durante o desenvolvimento do graduando, por isso, acaba sendo muito frequente a ocorrência de acidentes e/ou exposição a agentes biológicos durante as práticas, mesmo sob supervisão de profissionais. Foram entrevistados 11 enfermeiros docentes questionados sobre a concepção de biossegurança para estes. Surgiram três concepções, a primeira, sobre biossegurança como sinônimo de EPI; a segunda, associada a medidas mais amplas de proteção, que compreende EPI, Equipamentos de Proteção Coletiva (EPC) e promoção de um ambiente de trabalho seguro; e a terceira associa a biossegurança como uma forma de segurança da vida. Ressalta-se a necessidade de a Biossegurança ser mais bem exposta em cursos técnicos e universidades da área da saúde, favorecendo um maior senso crítico nesses profissionais (PEREIRA APDS, VERISSIMO TDC, 2019; RIBEIRO G, et al., 2019).

Uma pesquisa realizada com os estudantes de odontologia do último período da faculdade, mostrou que $100 \%$ dos participantes da pesquisa consideravam-se informados e cientes sobre a importância da biossegurança, reconhecendo inclusive os riscos que existem em suas atividades clínicas diárias. Todavia, apesar da noção do risco de infecção cruzada a que estão expostos, eles foram analisados durante a execução de alguns procedimentos, sendo observados que alguns acadêmicos negligenciavam procedimentos básicos de prevenção. Desse modo, nota-se que, embora os acadêmicos entrevistados afirmem bons conhecimentos acerca de biossegurança, muitos não aplicam esses conhecimentos durante as práticas. Portanto, o fato de ter conhecimento sobre o assunto, nem sempre isso vai proteger os profissionais e os estudantes de se acidentarem no ambiente de trabalho (NEVES HCC, 2011; RISSO M, RIBEIRO MC, 2003).

Em um hospital universitário no Rio de Janeiro, foi registrado um aumento de ocorrência de acidentes no período de 2008 a 2010, muitos deles, durante o atendimento ao paciente e a maioria ocorridos em profissionais com até 5 anos de trabalho. Nesse público detectou-se que 4,5\% dos entrevistados estavam sem EPI ou usando inadequadamente, resultando a exposição de materiais biológicos perigosos. Novamente revela-se a necessidade de um treinamento continuado de biossegurança na área da saúde (LAPA AT, et al., 2016; PEREIRA, EAA, et al., 2018)

Ao analisar a microbiota em unidade formadora de colônia (UFC), em bolsos e pulsos de quarenta e cinco jalecos distribuídos entre estudantes do curso de farmácia, biomedicina e ciências biológicas, destacou-se que os estudantes do curso de farmácia possuem uma maior quantidade de UFC na manga e bolso em comparação aos demais, sendo que, cerca de $20 \%$ desses estudantes não possuem consciência quanto ao uso do jaleco. Somado a isso o pesquisador Wiener-Well, citado em 2011, salientou em seus estudos que, ainda com um processo de higienização frequente, $63 \%$ dos jalecos dos profissionais de saúde observados apresentaram contaminação com bactérias potencialmente patogênicas, portanto, devem ser utilizados apenas em setores específicos e com higienização adequada (KAISER TL, et al., 2016). 
Corrêa LBD, et al. (2017) colocaram que entre janeiro de 2010 e dezembro de 2015, o total de profissionais de saúde que sofreram acidente de trabalho com exposição a material biológico no Estado do Maranhão foi de 1.919 casos, dos quais $41,34 \%$ utilizavam os EPI corretamente, enquanto que $58,66 \%$ admitiam a falha. $O$ que demonstra que o uso dos EPIs não garante $100 \%$ de proteção contra os acidentes perfurocortantes, e que para que o seu fator de proteção seja otimizado, é necessário que os profissionais saibam manuseá-los de forma correta

Outra grande preocupação relacionada com os acidentes de trabalho é a subnotificação de informações e a falta de seguimento clínico-laboratorial dos profissionais envolvidos nos acidentes. O estudo realizado por Sardeiro TL, et al. (2019) analisou os registros de acidentes com materiais biológicos entre 2006 e 2016, constatando que foram notificados 8596 casos no Estado de Goiás, depois da aplicação dos critérios de exclusão, restaram dois mil cento e quatro casos para a análise, sendo que destes que foram excluídos, mais de $45 \%$ eram devido a falta de informações nas fichas de notificação. Foi observado que $41,3 \%$ dos envolvidos em acidentes abandonaram o acompanhamento clínico-laboratorial.

O acompanhamento clínico-laboratorial após a ocorrência de acidentes de trabalho com exposição a materiais biológicos é de seis a doze meses. Inicialmente o paciente fonte deve ser submetido a teste rápido para a avaliação de possíveis doenças que podem ser transmitidas pelo contato com sangue, com HIV, HBV e HCV. Caso o resultado do teste rápido seja positivo, o acidentado deve iniciar quimioprofilaxia para a doença em específico e continuar com o seguimento clínico, caso seja negativo, deve continuar com o acompanhamento médico. O acompanhamento deve ser ambulatorial e dura de 6 a 12 meses, o profissional deve receber atendimento no momento do acidente, com 1 mês, com 3 meses, com 6 meses e com 12 meses, a depender do caso em questão. Caso não ocorra soroconversão, o paciente é liberado do serviço de saúde, caso ocorra a soroconversão, o paciente deve ser encaminhado para atendimento com médico infectologista (CAIXETA RB, BARBOSA-BRANCO A, 2005; GUIMARÃES HV, et al., 2011; RIFFEL FA, 2015).

Um grave problema que é bastante frequente nos ambientes de trabalho é a subnotificação desses acidentes, seja por falta de conhecimento dos profissionais da sua necessidade ou por medo de sofrer repreensão no ambiente de trabalho ou mesmo por ser um processo demorado. Para a notificação do acidente é necessário preencher a Comunicação de Acidente de Trabalho (CAT). É necessário que ocorra a conscientização dos profissionais de saúde sobre a importância de realizar o preenchimento correto da CAT, pois este é um instrumento utilizado pelo Ministério da Saúde para trabalhar na prevenção dos acidentes de trabalho (OLIVEIRA AC, GONÇALVES JÁ, 2009; TEIXEIRA CS, et al., 2008).

\section{CONSIDERAÇÕES FINAIS}

O presente estudo enfatiza a importância do uso adequado dos EPI no âmbito da saúde, reconhecendo a relevância da minimização dos riscos ocupacionais e dos acidentes a estes relacionados. Associa-se a significativa negligência dos profissionais quanto ao uso dos EPI a fatores como a falta de conhecimento e capacitação, a ausência de recursos necessários, o comodismo gerado pela autoconfiança de procedimentos rotineiros e até mesmo o desinteresse. Dessa maneira, é de grande valor a busca por alternativas que visam conscientizar profissionais de saúde quanto a biossegurança, desde a formação e durante todo o período de vida profissional ativa, com a educação continuada.

\section{REFERÊNCIAS}

1. ANDRADE GB, et al. Biossegurança: fatores de risco vivenciados pelo enfermeiro no contexto de seu trabalho/Biosafety: risk factors enhanced by the nurse in their work context. Revista de Pesquisa: Cuidado é Fundamental Online, 2018; 10(2): 565-571.

2. ARANTES DC, et al. Biossegurança aplicada à odontologia na Universidade Federal do Pará, cidade de Belém, estado do Pará, Brasil. Revista Pan-Amazônica de Saúde, 2015; 6(1): 8-8.

3. CAIXETA RB, BARBOSA-BRANCO A. Acidente de trabalho, com material biológico, em profissionais de saúde de hospitais públicos do Distrito Federal, Brasil, 2002/2003. Cad. Saúde Pública, 2005; 21(3): 737-746.

4. CORREAA LBD, et al. Fatores associados ao uso de equipamentos de proteção individual por profissionais de saúde acidentados com material biológico no Estado do Maranhão. Revista Brasileira de Medicina do Trabalho, 2017;15(4): 340-349.

5. DIAS ACB, et al. Adesão ao uso dos Epi's pela equipe de enfermagem no ambiente hospitalar: causas da resistência. 2015. 
6. DIAS MAC, et al. Acidente ocupacionais com exposição a material biológico: retrato de uma realidade. Medicina (Ribeirão Preto); 2012; 45(1):12-22.

7. GARCEZ GO, et al. Uso de EPI's pelos profissionais de saúde da atenção básica de um município de Goiás. Revista de Divulgação Científica Sena Aires, 2019; 8(4): 418-426.

8. GUIMARÃ ES HV, et al. Guia prático para avaliação e condutas frente aos acidentes com exposição a fluidos biológicos. 2011.

9. KAISER TL, et al. Avaliação da contaminação microbiana em jalecos de estudantes da área da saúde. Sa Bios-Revista de Saúde e Biologia, 2016; 11(1): 41-47.

10. LAPA AT, et al. Manuseio e descarte de perfurocortantes por profissionais de enfermagem de unidade de terapia intensiva The handling and disposal of sharps Among nursing staff of na intensive care unit. Revista de Pesquisa: Cuidado é Fundamental Online, 2017; 9(2): 387-392.

11. LEITE CJ, et al. Infecção cruzada entre laboratório de prótese e consultório: revisão de literatura. 2019.

12. NEVES HCC, et al. Segurança dos trabalhadores de enfermagem e fatores determinantes para adesão aos equipamentos de proteção individual. Rev. Latino-Am. Enfermagem, 2011; 19(2).

13. REZENDE KCAD, et al. Risco de exposição a material biológico em unidades de saúde da atenção primária à saúde. Revista de Enfermagem da UERJ. 2016;24:1

14. RIBEIRO G, et al. Concepção de biossegurança de docentes do ensino técnico de enfermagem em um estado do sul do Brasil. Trabalho, Educação e Saúde, 2015;13(3): 721-737.

15. RIBEIRO G, et al. Práticas de biossegurança no ensino técnico de enfermagem. Trabalho, Educação e Saúde. 2016;14:871-88.

16. RIBEIRO S, et al. Caracterização de acidente com perfurocortante de material e percepção de equipe de enfermagem. 2009. Cogitare Enfermagem, 14 (4), 660-666.

17. RISSO M, RIBEIRO MC. Equipamentos de Proteção Individual. In Silva A, Biossegurança em odontologia e ambientes de saúde. 2 ed. Cone editora; 2003: 67.

18. RIFFEL FA, et al. Análise epidemiológica de acidentes com material perfurocortante ocorridos com a equipe de enfermagem em um hospital na cidade de Santa Rosa/RS. Saúde Integrada - Biomedicina 2015.

19. RODRIGUES PS, et al. Acidente ocupacional entre profissionais de enfermagem atuantes em setores críticos de um pronto-socorro. Escola Anna Nery, 2017; 21(2).

20. SARDEIRO TL, et al. Acidente de trabalho com material biológico: fatores associados ao abandono do acompanhamento clínico-laboratorial. Rev. esc. enferm. USP, 2019; 53.

21. STANGANELLI, NC, et al. A utilização de equipamentos de proteção individual entre trabalhadores de enfermagem de um hospital público. Cogitare Enfermagem, 2015; 20(2).

22. SILVA TR, et al. Acidente com material perfurocortante entre profissionais de enfermagem de um hospital universitário. Revista Gaúcha de Enfermagem, 2010; 31(4), 615-622.

23. SOUSA AFL, et al. Representações sociais da Enfermagem sobre biossegurança: saúde ocupacional e o cuidar prevencionista. Revista Brasileira de Enfermagem, 2016; 69(5): 864-871.

24. PEREIRA APDS, VERISSIMO TDC. Adesão do uso de epi da equipe de enfermagem em um município da Amazônia legal. 2019.

25. PEREIRA EAA, et al. Motivações para mudança nas ações os profissionais de enfermagem após exposição acidental a material biológico. Revista de Pesquisa: Cuidado é fundamental online, 2018; 10(2): 534-541.

26. PINA E. O uso de luvas na prestação de cuidados de saúde. Rev. Nursing. 2010; 214: 28-33

27. OLIVEIRA AC, GONÇALVES JA. Incidência de acidentes com material perfurocortante entre alunos de graduação em ciências da saúde. Cienc Cuid Saude, 2009; 8(3):385-392.

28. TEIXEIRA CS, et al. Medidas de prevenção pré e pós Medidas de prevenção pré e pós-exposição a exposição a acidentes perfurocortantes na prática odontológica acidentes perfurocortantes na prática odontológica. Rev. odonto ciênc. 2008; 23(1):10-14. 